\title{
Smart city: automatic reconstruction of 3D building models to support urban development and planning
}

\author{
Nina Danilina ${ }^{1, *}$, Mihail Slepnev ${ }^{1}$, Spartak Chebotarev ${ }^{2}$ \\ ${ }^{1,2}$ Moscow State University of Civil Engineering, Yaroslavskoe shosse, 26, Moscow, 129337, Russia \\ ${ }^{2}$ OOO "Sigma Metrix", 105064, Zemlyanoy val,.34A, str.1, Moscow, RF
}

\begin{abstract}
The spread of smart city technologies dictates the need to develop new methods in the field of urban planning and design. The article devotes to the question how to find new approaches to make digital urban project according to the BIM- technology. We analyze the opportunities of automated design technology BuildingReconstruction 2018 that allows constructing 3D models of landscapes, urban territories and infrastructure objects. Their distinctive feature is their high accuracy of details. We consider methods and tools for creating such models. There is the description of a 3d-model and composing information layers. On the base of the analysis of the model's capabilities, we offer various directions how to use them for solving urban planning problems addressed to the examples of their existing implementation.
\end{abstract}

\section{Introduction}

The use of smart city technologies in urban design and planning requires clear understanding of required IT support solutions which should provide adequate development platform to the urban application domains. The reason is not only the huge amount of heterogenous and interconnected data but the inherently multi-disciplinary character of the application tasks (in-door and out-door navigation, air contamination, flooding, home-land security, property taxation, BIM, utilities \& communication networks management, etc.) and non-trivial interoperability challenges.

The article addresses the technical requirements to such software (SW) platforms, of data acquisition and processing techniques as well as practical large-scale implementations of semantic 3D modelling of city buildings. The issues of automatic diagnosis of most frequent modelling mistakes, and publicly available templets for domain application SW are also briefly discussed.

As a cornerstone of the required development platform the CityGML format was chosen [1]. This format was accepted by the International Open GIS Consortium (OGC) for semantic representation of $3 \mathrm{D}$ models of landscapes, urban territories, and city infrastructure objects, together with the Application Domain Extension.

\footnotetext{
Corresponding author: nina_danilina@mail.ru
} 
Modern urban planning sets high quality standards to the urban environment design and planning. The concept of smart city offers an appealing approach to the fusion of different IT techniques aimed at optimization of the territorial management and urban development. City 3D modeling and simulation is one of such advanced techniques which provides an unbiased and highly accurate visual representation of the actual use of an urban area together with powerful tools for the development of applied specialist applications on top of comprehensive spacial datasets. That helps assessing the architectural and planning solutions of a territory for the support of organizational and managerial decisions concerning sustainable development plans.

A good 3D modelling technology which is going be used in Smart City development environment must comply to an officially accepted international standard (e.g., ISO 19100) or to a widely used unofficial format with rich interoperability features. Most important, the format must be open and well documented. The minimum requirements to such a format should be as follows:

1. It has to be able to represents all relevant topographic object types of a city (buildings, vegetation, water, terrain, traffic, etc.).

2. It must represents city objects with $3 \mathrm{D}$ geometry, $3 \mathrm{D}$ topology, and appearance.

3. It should support unlimited hierarchical structure of features (georeferenced objects) and their components.

4. It should fully support user-definable and user-extendable textual attributes associated with city objects, including hyperlinks to the outside world.

5. Its structure should support adding new user-defined object types and provide for application domain extensions.

6. It should be excessively rich in semantics compared to pure 3D graphics and 3D map formats.

7. Should support sophisticated ad-hoc queries, simulations, and analyses.

In addition, the mechanisms for data storing and access should support data tyling or a similar approach.

At last but not the least, the development environment should cover he whole digital surface model of the territory (digital terrain model plus everything of interest on it) with known two-way interfaces to every single object, i.e., the objects actually must be able to communicate with each other. So far this basic element of the IoT (Internet of Things) was not even described in literature. Nevertheless, that shows the perspectives we should expect from this amasing field of applied sciences.

\section{Methods}

CityGML is the Open Geospatial Consortium OGC ${ }^{\circledR}$ encoding standard for the representation, storage and exchange of virtual $3 \mathrm{D}$ city and landscape models, [1]. CityGML models both complex and georeferenced 3D vector data along with the semantics associated with the data. In contrast to other $3 \mathrm{D}$ vector formats, CityGML is based on a rich, general purpose information model in addition to geometry and appearance information. That feature allows one to use the standard as a development platform for smart city applications.

For the fields of urban design and planning, targeted application areas (domains) explicitly include:

1. urban and landscape planning,

2. architectural design,

3. tourist and leisure activities,

4. $3 \mathrm{D}$ cadastres, 
5. environmental simulations,

6. vehicle and pedestrian navigation.

These are the typical Application Domain Extensions (ADE): specific "hooks" in the CityGML schema which allow one to define application specific extensions, for example for noise pollution simulation, or to augment CityGML by properties of the Building Information Model Standard (BIMS) [2,3].

Features of CityGML:

- Geospatial information model for urban landscapes

- GML3 representation of 3D geometries, based on the ISO 19107 model

- Representation of object surface characteristics (e.g., textures, materials)

- Classes of city objects:

- Digital Terrain Models as a combination of nested triangulated irregular networks, etc.

- Sites (currently buildings, bridges, and tunnels)

- Vegetation (areas, volumes, and solitary objects with vegetation classification)

○ Water bodies (volumes, surfaces)

- Transportation facilities (both graph structures and 3D surface data)

- Land use (representation of areas of the earth's surface dedicated to a specific land use)

○ City furniture

○ Generic city objects and attributes (could be user-definable objects).

Each object (or a group of objects) may have a certain Level of Detail (LoD):

$\circ$ LoD0 - regional, landscape

○ LoD1 - city, region (solid prisms with flat roofs, without wall openings and other detail)

- LoD2 - city districts, (as in LoD0 but with roof details)

○ LoD3 - architectural models (outside), landmarks (as LoD2 plus wall openings)

○ LoD4 - architectural models (as LoD3 plus interior walls and other details).

\section{Implementation}

Probably the most known software solutions fully compliant with the CityGML standard are the SW packages from virtualcitySYSTEMS, GmbH (Berlin, Germany). See [4] for details. The SW packages developed and owned by virtualcitySYSTEMS are becoming more and more popular among the urban development practitioners for its innovative and very efficient performance in urban 3D modelling. For us, probably most appealing features of the solutions were almost unlimited size of the modeled area, very high speed of automatic building reconstruction modules, and surprisingly low building modelling error rate.

The Building Reconstruction SW module (BREC) proved to be a very effective tool for automatic building geometry reconstruction, with or without additional manual model editing when needed. The BRec performance is amazingly high: it may build tens of thousands of building models within a working day, in unattended mode, on quite moderate office computers. The error rate (\% of poorly recognized models or hidden errors) is within few percent $(0.17 \%-1.6 \%)$ of the total buildings number when the input dataset is good enough.

A city model created with the use of BREC may consist of hundreds of thousand georeferenced buildings sitting on top of a digital terrain model. The text (semantic) attributes 
attached to each building model allow one to use such a model as the information support base for territorial management solutions, to be used in the smart city development.

Other SW modules of the virtualcitySYSTEMS offerings provide for the models storing, exchange, and publishing. That includes the Database Management System ("3D City DB") running on top of Oracle (commercial licensed SW) or PostgreSQL (public, free of charge).

Models created with BREC may be exported to other popular formats (e.g., $\mathrm{KML} / \mathrm{COLLADA} / \mathrm{gITF}$ with various display options and automatic tiling for Google Earth and Cesium (WebGL).

The Project was to explore the effectiveness of virtualcitySYSTEMS software in largescale automatic building geometry reconstruction and texturing for the use in urban design and development when implementing the smart city concepts. The distinctive feature of work was its large scale: over 200'000 models of existing buildings were generated in a purely automatic mode, and 50'000 models were manually edited.

For such a problem size and quite a short time span (ca 6 months), to manually build over 250'000 models is just not possible. So, the main target was to compare the effectiveness of a purely automatic model generation with the manual "beautification" of automatically restored models. Another task was to try to classify modelling errors found and get a preliminary estimate of the error rate.

Here we provide just preliminary though correct results; detailed error propagation analysis as well as the effectiveness of the automated diagnostics is still in the process.

Data acquisition, postprocessing and 3D modelling

For 3D building reconstruction in automated mode, the BREC SW was used, followed by the texturing stage with the use of Urban GRID Texturiser from UVM GmbH (Klosterneuburg, Austria, www.uvmsystems.com, www.citygrid.at ). The CityGML was used as the internal format for the exchange between BREC and Texturiser. The finished models were downloaded into 3DcityDB (Oracle).

Data acquisition starts with aerial photography and (optionally) aerial laser scanning. The photography was performed with digital nadir and oblique cameras (to get 3D images) mounted as Maltese cross on a fixed-wing airplane. The images have $35-75 \%$ of fore-andaft (forward and backward along the plane trajectory) overlap, and 30-60\% side overlap, depending on the plane speed and height. If there are not so many occlusions and the height is around 1'000 m., these imaging parameters normally are quite good for reconstruction of $3 \mathrm{D}$ geometry of walls and roofs as well as for the texturing of visible surfaces. Of course, the camera was a professional digital aerial optical system.

Aerial LiDAR (Light Identification Detection and Ranging) scanning provides the so called "point cloud of laser reflections" which consists of the image set of all points reflected by the surface and registered by the scanner receiver. The scan quality is normally measured as the average density of the cloud in the number of dots per one sq. m. For the modelling purposes, 1 to 40 dots per sq. $\mathrm{m}$. is enough depending on the task. We got ca 4-6 points per sq. $\mathrm{m}$.

These raw data were then processed at the photogrammetric office to get the input data for 3D modelling. These data are:

-Digital terrain model (DTM) covering the whole territory ca 1'000 sq. km.),

-Digital surface models (DSM) for the built-in areas

- Orthophotomaps, and

-Building footprints (vector contours) built manually using the orthophotomaps.

Next, BREC extrudes the building footprints from the DTM up until they hit the DSM (actually, the building roof). The roof shape is defined as the best fit to an internal catalogue template. This stage produces the geometry of LoD2 models.

A few actual numbers showing the SW efficiency: 
-Automatic building reconstruction: Performance rate: ca 10'000 per hour, Follow-up manual editing: ca 150 buildings per day per person.

-Automatic 3D geometry modelling: Geometry errors (non-planar walls (caused by bad footprints): $1.5 \%$, wrong building height (bad DSM due to tree shadowing the roof): $0.17 \%$, wrong roof shape (too few roof surfaces): ca $45 \%$ ).

-Automatic models texturing: no errors found yet.

All textured models with fixed errors were downloaded into Oracle structures.

The overall performance of BREC SW proved to be really high though the internal AD processes still require some more attention. Nevertheless, the SW is a very robust, efficient, and predictable tool for automatic reconstruction of LoD2 geometry, seemingly for really big input datasets.

As to the scene generalization, the simultaneous representation of LoD1 and LoD2 features goes very smooth and smart.

Lastly, object attributes, both plane text and hypertext, work fast and correct at bandwidth of $100 \mathrm{MHz}$. We tested live links to State databases and found no problem at all to connect and download alien data (address databases, land cadaster, 2D graphics in proprietary formats, MS Office files, etc.).

As the next stage of the Project, some automatic diagnostics (AD) and classification of errors experiments on this quite representative sample of actual data is being carried out. First step is to test some algorithms considered in $[5,6]$. After that we'll start to incorporate some ADEs for urban development applications (energy consumption, noise contamination, and air pollution dissemination). Integration with BIM structures and compiling a BIM $\mathrm{ADE}$ [4] is very desirable but is expected to start only sometime in the H1 of the year 2019.

The following issues constitute the main scientific and technical content of the virtualcitySYSTEMS (vcS, for short) platform in context of the Smart City concepts:

- fusion of visual representation of urban environment with technical description of territories, objects, and infrastructure,

-establishing the interrelations between natural and technical features of territories, objects, and infrastructure,

-development of tools to formalize the relationships between the structure of the territory and features and functionality of its components.

Analysis of the technological process of creating 3D models in the vcS environment, as well as the functional characteristics of VCS software suite allows us to think about quite a wide range of urban tasks which hopefully may be solved. There are a number of applications of such 3D models. Consider a few of possible domain applications that represent the most pressing issues arising in urban planning and design.

Visualization of actual and designed spatial solutions of urban areas. The dynamic pace of urban areas development claims for a quick response to the taken urban decisions. The 3D models of territories described above could be considered as an extension of BIM models where the object is not a single building but the territory as a whole. Such a model is not just a picture, but an information platform modelling the external and internal conditions in the urban area. That conditions refer to the actual state of the existing landscape, vegetation, transportation and engineering infrastructure as well as the relationship with the external world.

On Fig. 1, a 3D model of a new residential district in Moscow is given. The level of detail of the model allows to present not only the spatial solution and interrelationships of its elements, but the parameters of the area main elements: roadways, network of sidewalks, exits to the urban road network, the length of passages, etc. It is essential that such a model can provide a qualitative basis for the design of buildings, providing actual information about the use of the territory, and thus it becomes the basis for BIM-modeling of individual objects. 


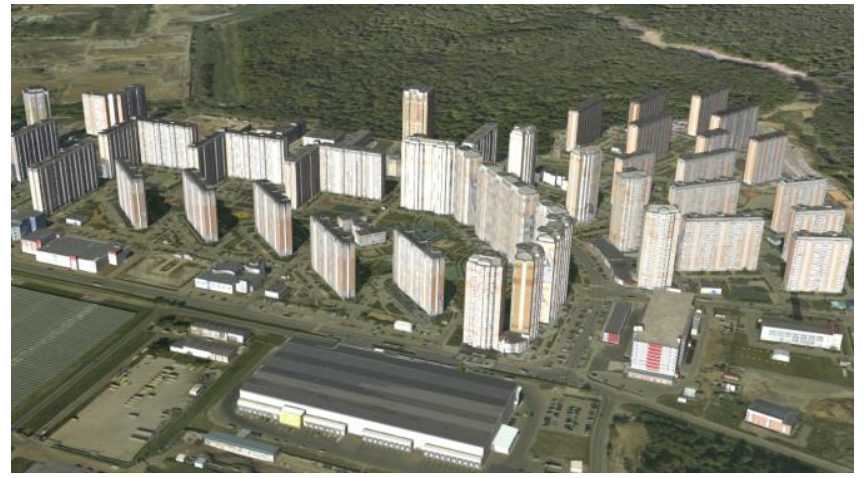

Fig. 1. 3D modeling of a typical residential area development, taking into account the transportation infrastructure and surrounding areas

Dynamic modeling of urban processes is one of the promising areas of urban science and practice. The detailed reality that the $3 \mathrm{D}$ model shows can not only mirror the static state but be also a basis for modeling dynamic processes in different areas of urban life. Examples of such processes may include:

-planning, organization, and management of intelligent transportation system,

-vehicle movement on-site;

-movement of pedestrians or non-motorized personal vehicles,

-escape routes in case of emergency, etc.

Such dynamic models are aimed at determining the relationship between the organization of the territory and the behavior habits of its users in order to improve the quality, safety and comfort of people's life being.

The town-planning analysis of the natural processes influencing the development of territories is necessary for the decisions making concerning its use. This area of application is particularly important for territories located in areas with specific climatic, landscape, and engineering conditions $[7,8]$.

This area of application of 3D models is of clear scientific and applied nature. On the one hand, it allows to assess the impact of natural factors on the environment. On the other, - it is a valuable material for research, uncovering the interdependencies and the development of universal methods of urban planning and design. Here are several possible areas of work:

- assessing bioclimatic factors, aeration, insolation, seismic hazard in the territory. Especially important for areas with extreme natural conditions. E.g., for the Russian

Federation this issue is particularly relevant in the Federal programs of development of Arctic or the Far East;

- mapping the spread of environmental pollution: noise, vibration, the air contamination;

- assessment of qualitative and quantitative characteristics of natural areas based on data from the digital model of vegetation, DTM and slopes, etc.

Information storage of large amounts of information. The ability to attach attribute information to model elements (Fig.2) opens up prospects for using the 3D model as a Big Data storage. Information can be of any nature, depending on the customer's information model and may be referenced to a specific object, area, territory, and region. Information may be abstract, not directly related to the appearance of the object or territory, e.g., it could be information about land users. 


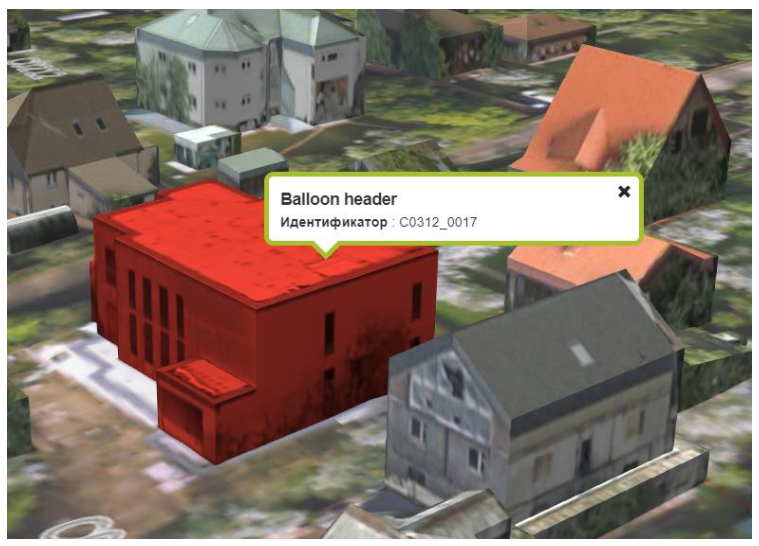

Fig. 2. 3D object model with attached attributes

It could also be a snapshot of an existing state. For example, to create a register of objects of cultural heritage, preservation of existing or reconstruc-tion of spatial planning and architectural solu-tions, the organization of the territory.

Thus, we can use this model as a visual image to store any information associated with it, and to preserve the history of its appearance for future generations. On Fig. 3, an example of the historical heritage is given.

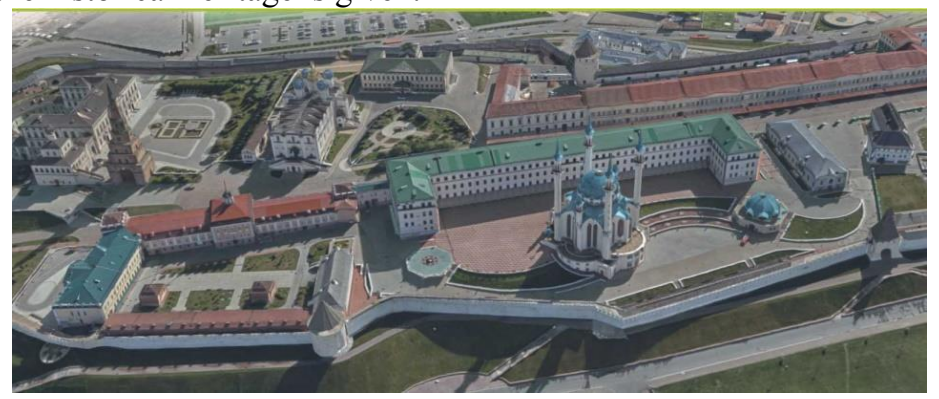

Fig. 3. 3D reconstruction of the historical territory (Kazan kremlin)

This project is a part of the research work "Experimental statistical research in the environment of computer-aided design BuildingReconstruction 2018", performed by the Urban Planning Department of Moscow State University of Civil Engineering under Research contract with "Sigma metrics" Limited, Moscow, Russia.

The theme of the introduction of 3D models in urban planning is one of the promising trends in the context of digitalization of cities.

Smart technologies are a tool to improve the quality, safety, permeability of urban environment. Currently, there are active discussions on how to implement smart technologies starting as early as at the stage of territorial planning of cities. In this aspect, the presented 3D modelling approach can be a step towards the development of comprehensive intellectual development platform for urban design and planning as a valuable part of the Smart City concept.

\section{Conclusions}

Modern technologies are changing the urban environment, the economic landscape, and social relations, create the opportunity to manage municipal farms at a qualitatively new level and create an active demand for new digital services in this area. In the field of urban development, many different initiatives are already being implemented, including an important step of creating the public 3D platforms that will be very helpful in solving 
various urban problems. The main purpose of the $3 \mathrm{D}$ model is to provide a close connection between smart city projects -- street video surveillance, public services, intelligent transport system and others, -- on the scale of cities and regions.intelligent transport system - and others on the scale of cities and regions.

\section{References}

1. Gröger, G., Kolbe, T. H., Nagel, C., Häfele, Encoding Standard. www.citygmlwiki.org, (2012)

2. Nagel, C., www.virtualcitysystems.de (2017)

3. Floros, G., Dimopoulou, E., The International Archives of the Photogrammetry, Remote Sensing and Spatial Information Sciences, V. XLII-2/W2, (2016)

4. Biljecki, F., Kumar, K., Nagel, C., Springer Int. Publ. , https://doi.org/10.1186/s40965018-0055-6, 2018.

5. Michelin, J-C., Tierny, J., Tupin, F., Mallet, C. and Paparoditis., https://wwwpequan.lip6.fr/ tierny/stuff/papers/michelin_ssg13.pdf. (2013)

6. Biljecki, F., Ledoux, H., Du X, Stoter, J., Soon, K.H., Khoo, V.H.S., ISPRS Ann Photogrammetric Remote Sens Spatial Inf Sci., IV-2/W1, pp. 13-22. https://link.springer.com/content/pdf/10.1186\%2Fs40965-018-0055-6.pdf. (2016)

7. Danilina N., Elistratov D. Transp. Res. Proc. doi: 10.1016/j.trpro.2017.01.034 (2017)

8. Danilina N. Mater. Sc. and Eng. (IOP Conf. s), V. 365, doi: 10.1088/1757$899 \mathrm{X} / 365 / 2 / 022002$ 